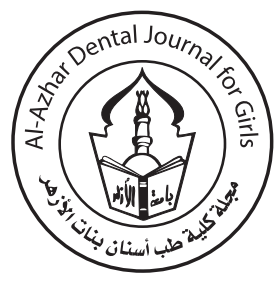

\title{
Volumetric Bone Change After Using Liquid Phase Concentrated Growth Factor in Open Sinus Lift Surgery: A Comparative Study
}

\author{
Nada B. Hanafy ${ }^{1 *}$, Nahed A. Abd Elmoniem ${ }^{2}$, Hatem H. Al Ahmdy ${ }^{3}$
}

Codex : 12/21.10

azhardentj@azhar.edu.eg

http://adjg.journals.ekb.eg

DOI: 10.21608/adjg.2021.38138.1281

Oral Medicine \& Surgical Sciences (Oral Medicine, Oral \& Maxillofacial Surgery, Oral Pathology, Oral Biology)

\section{KEYWORDS}

Liquid phase concentrated growth factor (LPCGF),

Biphasic calcium phosphate (BCP),

Maxillary sinus lift (MSL)

\begin{abstract}
Purpose: To evaluate of the volumetric bone change after using liquid phase concentrated growth factor in open sinus lift surgery .Material and methods 12 pneumatized maxillary sinuses in edentulous or partially edentulous patients were divided randomly into 2 equal groups; Group (A), augmented by alloplastic bone graft Biphasic Calcium Phosphate (BCP). Group (B), augmented with BCP mixed with liquid phase concentrated growth factor (LPCGF). Visual Analogue Scale (VAS) was used to assess pain and swelling. CBCT was used to assess the volume by measuring height $\times$ surface area of augmented bone immediate and 6 months postoperatively. Clinical follow up period was at $1^{\text {st }}, 2^{\text {nd }}$ day then, after one week postoperatively. Results 12 sinus , bilateral $71.4 \%$, unilateral $28.5 \%$, female $42.8 \%$, male $57.2 \%$ were subjected to sinus lift surgery. Mean age was 50y and average length of residual bone was 7 $\mathrm{mm}$. There was no significant difference between the two groups regarding primary wound healing with percentage of success $71.4 \%$. VAS scale representing swelling of $42.8 \%$ in group (A) and $28.5 \%$ in group (B). Pain score show decrease gradual till one week to be zero. In comparing between two groups, percentages of change in volume was highly significant $(\mathrm{p}=0.004)$ between two groups; in group (A) was $29.31 \pm 13.23$ while, in group (B) was $2.58 \pm 1.75$. Conclusion: Use of LPCG mixed with $\mathrm{BCP}$ bone graft to augment the sinus with open sinus lift surgery showed less bone resorption as it represented with low percentage of change in bone graft volume at 6 months postoperative .
\end{abstract}

- Paper extracted from master thesis titled "Volumetric bone changes after using liquid phase concentrated growth factor in open sinus lift surgery: A comparative study."

1. Dentist at Nuclear Materials Authority, MSc student, Department of Oral and Maxillofacial Surgery ,Faculty of Dental Medicine for Girls, AL-Azhar University, Cairo, Egypt.

2. Assistant professor of Oral and Maxillofacial Surgery Department, Faculty of Dental Medicine for Girls, AL-Azhar University, Cairo, Egypt.

3. Head of the Department of Oral and Maxillofacial Surgery, Faculty of Dental Medicine for girls, Al-Azhar University

* Corresponding author email: dr.nodybadr@gmail.com 


\section{INTRODUCTION}

Implant dentistry had been excellent treatment modality ever since its ingress into the field of modern dentistry. However, the requirement for the placement of an implant is the presence of an adequate alveolar bone. The quality and the quantity of the bone have to be sufficient for implant placement. Posterior maxilla often presents with insufficiently available bone for the placement of implants due to the pneumatization of maxillary sinus and resorbed ridge ${ }^{(1,2)}$. The initial implant stability is poor coupled with inadequate bone height for the implant length.

Several sinus augmentation procedures for implant placement have been introduced since the $1980 \mathrm{~s}^{(3,4)}$. Autogenous bone, allograft, xenograft, alloplastic bone, or combinations of them ${ }^{(5,6)}$. Autogenous bone is considered to be the gold standard, it creates another wound at the donor site. The other graft materials also have limitations, including risk of inflammation, insufficient bone regeneration, and increased overall cost. Accordingly, no graft material appears to be superior to the other ${ }^{(5)}$.

Some materials are used to enhance the effect of the graft material such as , platelet-rich plasma( PRP) ${ }^{(7)}$ was developed as first generation as early as the $1970 \mathrm{~s}^{(\mathbf{8})}$, which contain multiple growth factors ${ }^{(9)}$ Platelet-rich fibrin (PRF) was first reported in 2001 by Choukroun et al. as a second-generation platelet concentrate $^{(\mathbf{1 0 , 1 )}}$. It was used for both hard and soft tissue regeneration ${ }^{(11)}$. Liquid phase concentrated growth factor (LPCGF) is the latest generation of PRP, which was developed by Sacco in $2006^{(12)}$. It was reported that LPCGF are stronger and purer than PRP. Moreover, such growth factors that are centrifuged can continuously and slowly discharge for at least 7 to10 days. ${ }^{(13)}$ So, the aim of this study was to evaluate the effect of (LPCGF) on volume bone changes after added to BCP in sinus augmentation through open sinus lift surgery.

\section{MATERIAL AND METHODS}

Controlled and randomized clinical trial was conducted on 12 pneumatized maxillary sinuses at edentulous and partially edentulous patients need implant reconstruction, selected from the outpatient clinic of the Department of Oral and Maxillofacial Surgery, Faculty of Dental Medicine for Girls, Al-Azhar University and AL-Zahraa educational hospital, Cairo, Egypt. Protocol was approved by the National Research Centre and Al-Azhar University. Informed consent was obtained from all subjects involved.

The inclusion criteria: patients with pneumatized maxillary sinus of edentulous or partially edentulous patient need construction of implant. The alveolar ridge height is less than $5 \mathrm{~mm}^{(4)}$.Exclusion criteria: Systemic diseases interfere with bone healing, poor oral hygiene, acute sinusitis and heavy smoker $^{(5)}$

Patients were divided equally into 2 groups; Group (A), open sinus lift surgery was done on six pneumatized maxillary sinuses and augmented with BCP. Group (B), open sinus lift surgery were done on six pneumatized maxillary sinuses and augmented with BCP mixed with LPCGF. All patients were followed up clinically $1^{\text {st }}$ two days, then one week postoperatively and $\mathrm{CBCT}$ at immediate and 6 months postoperatively.

\section{Presurgical Preparation}

Demographic data were collected in patient chart, Panoramic X-ray for preliminary preoperative evaluation of the alveolar bone height and the sinus pneumatization, sinusitis and any pathologic lesions. Instruction of oral hygiene measure. CBCT (Scanora 3D Soredex, Helsinki, Finland) for 3D assessment of alveolus bone and surgical guide planning, designed and printed by CAD software (Ondemand 3D, Seoul, South Korea).

\section{LPCGF Preparation:}

$10 \mathrm{cc}$ venous blood collected in sterile tubes with $1 \mathrm{cc}$ citrate coat the wall of the tube. Sequential cen- 
trifuging protocol: $30 \mathrm{sec}$-acceleration, $2 \mathrm{~min}-2700$ rpm, 4min -2400 rpm, 4min - $2700 \mathrm{rpm}, 3 \mathrm{~min}-$ $3000 \mathrm{rpm}$, lastly, $36 \mathrm{sec}$ - deceleration. This results in 4 different phases ${ }^{(15)}$ from up to bottom were Serum, Fibrin buffy coat, CGF and red blood cells.

\section{Open Sinus Lift Surgical Procedure(Fig.1)}

Crestal incision with two vertical releasing incision, mucoperiosteal flap was elevated to expose the lateral sinus wall and allow the surgical guide to be easy adapted and seated over the bone, sinus opening was done at the exact position guided by surgical stent using the bone cutting piezosurgery tip to avoid perforation of the delicate sinus lining mucosa. Sinus lift kit was used to elevate the sinus lining in four directions to be ready for augmentation. Gel foam was added in all the cases of both groups before augmentation of $2.5 \mathrm{cc}$ of BCP (alloplastic graft consists of $60 \%$ hydroxyapatite and $40 \%$ b-tricalcium phosphate, has a $90 \%$ porous surface with 100-500 $1 \mathrm{~m}$ pores) in group (A) while in group (B); LPCGF was mixed with BCP.

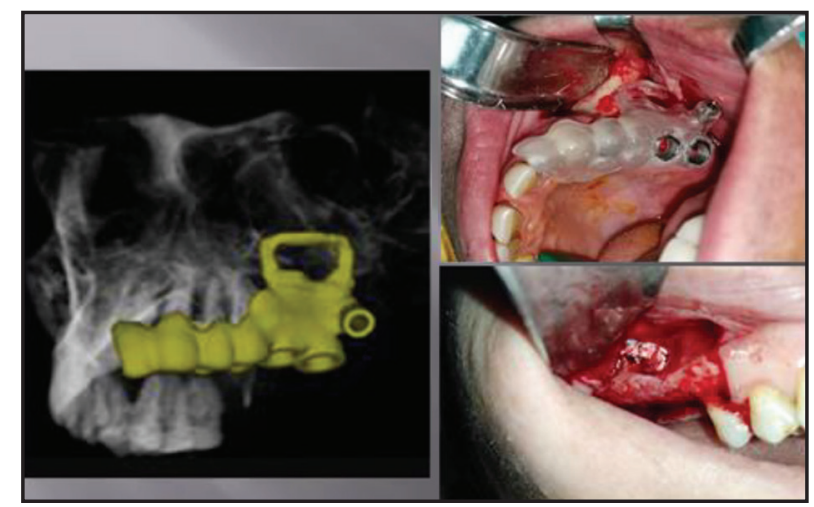

Figure (1) Sinus Lift Surgical Guid 3D Printing Design Used In Open Sinus Lift.

\section{Postoperative Clinical Assessment:}

Pain, swelling were evaluated by Visual Analogue Scale (VAS), in addition to wound dehiscence and/ or infection.

\section{Postoperative Radiographic Assessment:}

Volumetric bone graft was measured by area and linear measurements for each case immediately and 6 months postoperative. The median of three successful readings was calculated by the equation (Area of the graft $\times$ its height). Area was measured from axial cut by demarcation the graft outline including antro-posterior and mesio-distal dimension. The bone graft height was measured from lower to the highest point of the graft over alveolus from sagittal view.

\section{Statistical Analysis:}

All data were subjected to statistical analysis using descriptive and paired sample t-tests. Differences at $\mathrm{p}<0.05$ were considered significant. Calculations were performed using the SPSS (Statistical Package For Scientific Studies) for (Windows 20.0).

\section{RESULTS}

\section{Demographic Data}

A total of 12 sinus ( 5 patients bilateral $71.5 \%$, 2 patients unilateral $28.5 \%$ ), 3 females $42.8 \%, 4$ males $57.2 \%$ were subjected to sinus lift surgery. Age ranged from 40 to 60 years old. The average length of the residual bone were $7 \mathrm{~mm}$ and the average volume of bone graft was $1530 \mathrm{~mm}^{3}$

\section{Clinical Outcomes}

There was no significant difference between the two groups regard primary wound healing with percentage of success $71.4 \%$. However, $28.5 \%$ of cases showed wound dehiscence; one at each group. VAS scale representing swelling of $42.8 \%$ in group (A) and $28.5 \%$ in group (B). Pain score (5 in G(A) and 4 in $G(B)$ ) in $1^{\text {st }}$ two days after operation then decrease gradual till one week to be zero. 


\section{CBCT Outcomes}

\section{Grafted Bone Volume Measurement}

Table (1): Percentage of change in volume of bone graft along follow up period

\begin{tabular}{lcccc}
\hline & Group A & Group B & MW & P \\
\hline $\begin{array}{l}\text { Percentage of change }(\%) \text { in volume } \\
\text { from immediate to 6 months }\end{array}$ & $29.31 \pm 13.23$ & $2.58 \pm 1.75$ & 3.958 & $0.004 *$ \\
\cline { 2 - 4 } & $27.28(10.55-47.76)$ & $2.07(0.56-4.84)$ & & \\
\hline
\end{tabular}

MW= Mann Whitney $\quad P=P$ value $*$ statistically significant $p$-value $\leq 0.05$

Volume of grafted bone: In group (A), at immediate post-operative was $(2409.15 \pm 358.03)$ and at 6 months postoperative was $(1695.43 \pm 456.5)$ with $\mathrm{p}=0.002$. While, in group (B) at immediate postoperative bone volume was $(2257.47 \pm 560.38)$ and at 6 months postoperative was $(2209.1 \pm 573.81)$ with $\mathrm{p}=0.59$.

In comparing between two groups, percentages of change of bone volume was highly significantly ( $p=0.004)$ between two groups. Percentage of change in the volume of graft was $29.31 \pm 13.23$ in group (A) while, in group (B) was 2.58 \pm 1.75 .

\section{DISCUSSION}

Open sinus lift procedure allows bone augmentation of atrophied maxillary posterior region to increase not only the vertical bone height but also, medio-lateral and ant-posterior areas with sufficient amount of bone. Amount of bone graft material used on our study was about $2,5 \mathrm{cc}$ that equal to $2500 \mathrm{~mm}^{3}$ which cannot be applied by close method and thereby facilitating more than one implant placement.

In our study, CBCT was helpful in diagnosis and planning of the surgical guide that designed to use for two steps, first to demarcate the sinus opening and to allow implant placement without surgical flap at the end of follow up period. ${ }^{(14)}$ The guide demarcate the lateral sinus window outline at the exact site for grafting the area of missing teeth that allow placement of implant surrounding with sufficient amount of bone surrounding all over its length. In addition, the location of the implant site drilling in the accurate position and angulation without need to do another one that decrease cost and time..$^{(2,3)}$

The piezo-surgery tips for bone cutting was selected. It was used successfully for removing bone without perforating the sinus lining which was very delicate and thin in all the cases. ${ }^{(15,16)}$

Gel foam ${ }^{(17)}$ was used in all the cases before insertion of the graft material within the sinus as a protective or caution layer to allow retraction of sinus lining as the BSP has rough surface and is considerably large in size that may tear or perforate the sinus lining during its application.

$\mathrm{BCP}$ is an alloplastic grafting material thought to be a suitable and effective bone substitute for new bone formation in dental clinical application ${ }^{(18-20)}$.In this study, BCP was used as bone substitute grafting material. The BCP particles has a large particle size that provide adequate volume $\left(2500 \mathrm{~mm}^{3}\right)$ to overcome the severe deficiency at posterior maxilla as well as it has a $90 \%$ porous surface with 100 $500 \mathrm{~lm}$ pores which has the benefit to replace by new bone formation in spite of containing hydroxyl appetite. In addition, it thought to be suitable to be mixed with LPCGF that also proved to accelerate bone healing. LPCGF aids in bone proliferation which enhance soft tissues as well as bone healing ${ }^{(21)}$. 
CGF in liquid phase releases various growth factors such as Platelet-derived growth factor (PDGF), Transforming growth factor- $\beta 1$ (TGF- $\beta 1$ ) and $\beta 2$ (TGF- $\beta 2$ ), Fibroblast growth factor (FGF), Vascular endothelial growth factor (VEGF), Brain derived growth factor (BDGF) and Insulin-like growth factor (IGF) which stimulate cell proliferation and angiogenesis and stimulate osteoblast proliferation and differentiation. ${ }^{(22)}$

During this study the volume of bone was calculate as (cross section length $\times$ the area of each section) this method are more accurate due to it detect each point around the graft area and measuring of 3 successful reading and take their mean was also another way for accuracy.

In comparing between two groups, percentages of change in bone volume from immediate postoperative to end of follow up period was highly significantly ( $\mathrm{p}=0.004$ ), in group (A) was $29.31 \pm 13.23 \%$ while, in group (B) was $2.58 \pm 1.75 \%$. This result indicated that, LPCGF has an effective rule, in decreasing amount of bone resorption by $2.58 \pm 1.75$ and improve bone healing.

\section{CONCLUSION}

Use of LPCG mixed with BCP bone augmentation with open sinus lift showed less bone resorption as it represented with low percentage of change in bone graft volume at 6 months postoperative follow up period.

\section{REFERENCES}

1. Parra M, Olate S, Cantín M. Clinical and biological analysis in graftless maxillary sinus lift. J Korean Assoc Oral Maxillofac Surg . 2017;43:214-20.

2. Anbiaee N, Khodabakhsh R, Bagherpour A. Relationship between anatomical variations of sinonasal area and maxillary sinus pneumatization. Iran J Otorhinolaryngol. 2019; 31:229-34.

3. Ueno D, Banba N, Hasuike A, Ueda K, Kodama T. A Sinus Floor Reaugmentation Technique Around an Apically Exposed Implant Into the Maxillary Sinus. J of Oral Implantol. 2019;45:213-7.
4. Bathla SC, Fry RR, Majumdar K. Maxillary sinus augmentation. J Indian Soc Periodontol . 2018;22:468-73

5. Silva L, de Lima VN, Faverani LP, de Mendonça MR, Okamoto R, Pellizzer EP. Maxillary sinus lift surgerywith or without graft material? A systematic review. Int J Oral Maxillofac Surg. 2016;45:1570-6

6. Ali S, Bakry SA, Abd-Elhakam H. Platelet-rich fibrin in maxillary sinus augmentation: a systematic review. J Oral Implantol .2015;41:746-53.

7. Keestra JA, Barry O, Jong LD, Wahl G . Long term effects of vertical bone augmentation: a systemic review. J Appl Oral Sci. 2016; 24 :3-17.

8. Bos-Mikich A, de Oliveira R, Frantz N. Platelet-rich plasma therapy and reproductive medicine. J Assist Reprod Genet.2018;35: 753-56

9. Shahana CM, Subija KN . Platelet-Rich Fibrin: Role in Periodontal and Pulpal Regeneration .JMSCR . 2017; 5: 22447-52.

10. 10- Jagati A, Chaudhary R, Rathod S, Madke B, Baxi K, Kasundra D. Preparation of platelet-rich fibrin membrane over scaffold of collagen sheet, its advantages over compression method: A novel and simple technique. JCAS. $2019 ; 12: 174-8$

11. Fan Y, Perez K, \& Dym, H. Clinical Uses of Platelet-Rich Fibrin in Oral and Maxillofacial Surgery. J of Dent Clin North Am . 2020;64: 291-303

12. Miranda S, Costa MF ,Rebouças N, Ramos MT, Lessa DA, Alencar NX. Protocols for preparation of platelet rich plasma (PRP) in Quarter Horses. J of Pesq Vet Bras. 2019; 39:614-21

13. Jing Qiao, Jinyu Duan, Yong Zhang, Y. C. Changzhou Sun. The effect of concentrated growth factors in the treatment of periodontal intrabony defects. J of Futur Sci OA. 2016; 24: 2-4

14. Yan Q, Dong H, Su J, Han J, Song B, Wei Q, et al. A Review of 3D Printing Technology for Medical Applications. J of Engineering.2018;4:729-42

15. Gupta KK, Bathla S. Advanced implant surgery. Textbook of Periodontics. 1st ed. New Delhi: Jaypee Brothers Medical Publishers. 2017;1:636-46.

16. De Carvalho MA, Marques G, Trento GD, Padovan LE, Klüppel LE. utilização do sistema piezoelétrico em cirurgias bucais: indicações, vantagens e desvantagens. J of Dent \& Public Health. 2017;8:13-15 
17. Sahand S, Majid E, Elahe T, Anahid J, Rashid S, Hamed K. Using Absorbable Gelatin Sponge to Facilitate Sinus Membrane Elevation during Open Sinus Lift: Technical Notes and Case Series. JDMT.2019:8:1-7

18. De Oliveira PI, Poli PP, Maiorana C, De Vasconcelos IR, Schmidt LE, Colombo LT, et al. Evaluation of osteoconduction of biphasic calcium phosphate ceramic in the calvaria of rats: Microscopic and histometric analysis. J Funct Biomater. 2019;1:10 -13

19. Padalhin AR, Lee B. Hemostasis and Bone Regeneration Using Chitosan/Gelatin-BCPBi-layer Composite Material. JASAIO. 2019;65:620-27.
20. Nie L, Deng Y, Li P, Hou R, Shavandi A, Yang S. Hydroxyethyl Chitosan-Reinforced Polyvinyl Alcohol/ Biphasic Calcium Phosphate Hydrogels for Bone Regeneration. JACS Omega. 2020 5:10948-57.

21. Bernardi S, Mummolo S, Tecco S, Continenza MA, Marzo G. Histological Characterization of Sacco's Concentrated Growth Factors Membrane. Int J Morphol. 2017 ;35:114-9.

22. Wang L, Wan M, Li Z, Zhong N, Liang D. A comparative study of the effects of concentrated growth factors in two different forms on osteogenesis in vitro. J Mol Med Rep. 2019; 20:1039-48. 